\title{
Arthroscopic treatment of shoulder instability in professional athletes
}

\author{
Andrea Pantalone \\ Daniele Vanni \\ Matteo Guelfi \\ Michele Di Mauro \\ Michele Abate \\ Vincenzo Salini
}

Department of Medicine and Science of Aging, University "G. d'Annunzio" Chieti - Pescara, Chieti, Italy

Corresponding author:

Michele Abate

Department of Medicine and Science of Aging,

University "G. d'Annunzio" Chieti - Pescara

Via dei Vestini 31

66013 Chieti, Italy

E-mail:m.abate@unich.it

\section{Summary}

Background: Post-traumatic shoulder instability is a common disease, especially in sportsmen. If inadequately or late treated, it may be responsible for an articular biomechanics alteration, with serious problems. This is much more obvious for professionals athletes, because corporate and market needs force them to a premature return to sport. The purpose of this retrospective study is to evaluate if arthroscopic approach may be better than the open one and allowing a shoulder function rapid recovery, with fast return to sport.

Materials and methods: From January 2003 to January 2014, 46 professional athletes underwent surgical treatment for post traumatic shoulder instability. Two groups were made: 25 athletes treated arthroscopically and 21 treated with open approach. Patients were followed up from 36 to 92 months, according to Rowe Score for Instability and VAS scoring system.

Results: Patients in the open group returned later than the first one to sport. Moreover, pain in postoperative period and during rehabilitation, was lower in the arthroscopic group. There was only one recurrence in the open group.

Conclusions: Arthroscopic surgical approach appears to be excellent in shoulder instability management, reducing recovery time, allowing a faster return to sport, with less pain, if compared with open surgery.

Level of Evidence: III, a case control-study.

KEY WORDS: arthroscopy, athletes, instability, open surgery, shoulder.

\section{Introduction}

The glenohumeral joint is equipped with static and dynamic stabilizers (glenoid labrum, glenohumeral ligaments, articular capsule and rotator cuff muscles), it has a large range of motion (ROM) and it is equipped for high sports performance, but it is simultaneously vulnerable and exposed to possible injuries. Shoulder instability treatment requires special attention in professional athletes, as external pressures about recovery times and rapid return on highperformance gaming force a premature recourse to sport. Several surgical techniques (arthroscopic or open) have been proposed to stabilize these shoulders. The arthroscopic procedure seems to be associated with a significant risk of recurrence in athletes ${ }^{1}$. In fact, Bessière et al. declared that patients who had the open Latarjet procedure had less recurrent instability and better Rowe scores over a mean 6 -year follow-up ${ }^{2}$. At the same time, Blonna et al. stated that arthroscopic stabilization using anchors provided better return to sport compared with the open Bristow-Latarjet procedure in the population studied $^{3}$. The purpose of our study is to evaluate if arthroscopic approach may be better than the open one, reducing recovery time and allowing a shoulder function rapid recovery, with fast return to sport.

\section{Materials and methods}

From January 2003 to January 2014, a series of 46 professional athletes was surgically treated for post traumatic shoulder instability. From April 2016, each Author is required to conduct his research ethically according to international standards and as required by the Journal as described in Padulo et al. ${ }^{4}$. The case study consists of 38 men and 8 women in an age range from 18 to 35 years (13 soccer players, 11 basketball players, 10 martial arts fighters, 8 motorbike riders and 4 rugby players), with an average age of 25 years. Of these patients, 21 underwent open Bankart repair and 25 were arthroscopically treated. 
Surgical treatment consists of a capsulolabral reconstruction with anchors. Procedure choice occurred randomly. In the present study patients with Atraumatic, Multidirectional, Bilateral, Rehabilitation, Rotator interval reconstruction, Inferior capsule tightening (AMBRII) or Acquired, Instability, Overstressed, Surgery (AIOS) type of instability, bony Bankart lesion or previously undergone surgery for shoulder instability were not included.

Physical examination showed shoulder girdle muscles weakness and shoulder ROM reduction, with difficult in arm abduction and external rotation. Apprehension test, Fulcrum test, and Relocation test were preoperatively performed and the sliding of the humeral head was graded according to the Society of American Shoulder and Elbow Surgeon Scale (15 Grade I, 40 Grade II and 21 Grade III). Standard radiological examination ${ }^{5}$ was carried out on all patients and followed up by M.R.I. or CT scan (to exclude unknown associated fractures also). In patients treated by open approach (mean incision $6 \mathrm{~cm}$ ) was performed a capsulolabral repair with three metal anchors, placed at 6, 3 and 2 o'clock. The suture of the capsule was made with the arm at $45^{\circ}$ of abduction, $15^{\circ}$ of flexion and neutral rotation, under a slight traction. Subscapularis was then sutured anatomically after making sure that the arm could reach $30^{\circ}$ of external rotation.

Both surgical procedures were performed in beach chair position, a semi-sitting position that allows an easy access through the portals, inside of the joint, allowing at the same time to perform shoulder passive movements, in order to conduct a diagnostic (Fig. 1) and then naturally, a reparative arthroscopy (Figs. 2, 3). Traditional anterior, anterosuperior and posterior portals were used.

Capsulolabral tissues were mobilized up to the line of 6 o'clock and the glenoid anterior edge was refreshed using a shaver. Capsulolabral complex was repaired using 3 metal anchors. For postoperative care, an abduction shoulder brace at $15^{\circ}$ for 4 weeks was advised. Subsequently, a cautious physiotherapy was appropriate, aimed at R.O.M. gradual recovery, using hydrokinesistherapy also. In the later stages of rehabilitation time and during the game, kinesiotaping was used ${ }^{6}$. It was very important both avoid external rotation in the early stages and recover, as soon as possible, sports specific gestures (active and passive), so that the normal range of motion and muscle strength could be recovered. In the postoperative period, as done at the clinic and described in the literature ${ }^{7}$, it was beneficial perform an assessment postsurgery M.R.I. to ascertain the correct positioning of the anchors and of the glenoid labrum and to evaluate the possible presence of intracancellous edema and/or joint effusion. The clinical results were evaluated using the Rowe Score for Instabili$t^{8}{ }^{8}$. The severity of pain in the early postoperative period was assessed using a visual analogue scale (VAS) on a numeric scale of $0-10$. The mean follow-up period was 64 months (range 36 to 92 months). The results were evaluated with a $95 \%$ confidence interval, taking $\mathrm{p}<0,05$ as the significative level.

\section{Results}

Mean duration of surgical operation was 1 hour and a half for open surgery, and 1 hour for arthroscopic repair. Mean Rowe score was 90 in the open surgery group, and 95 in the arthroscopic repair group. Based

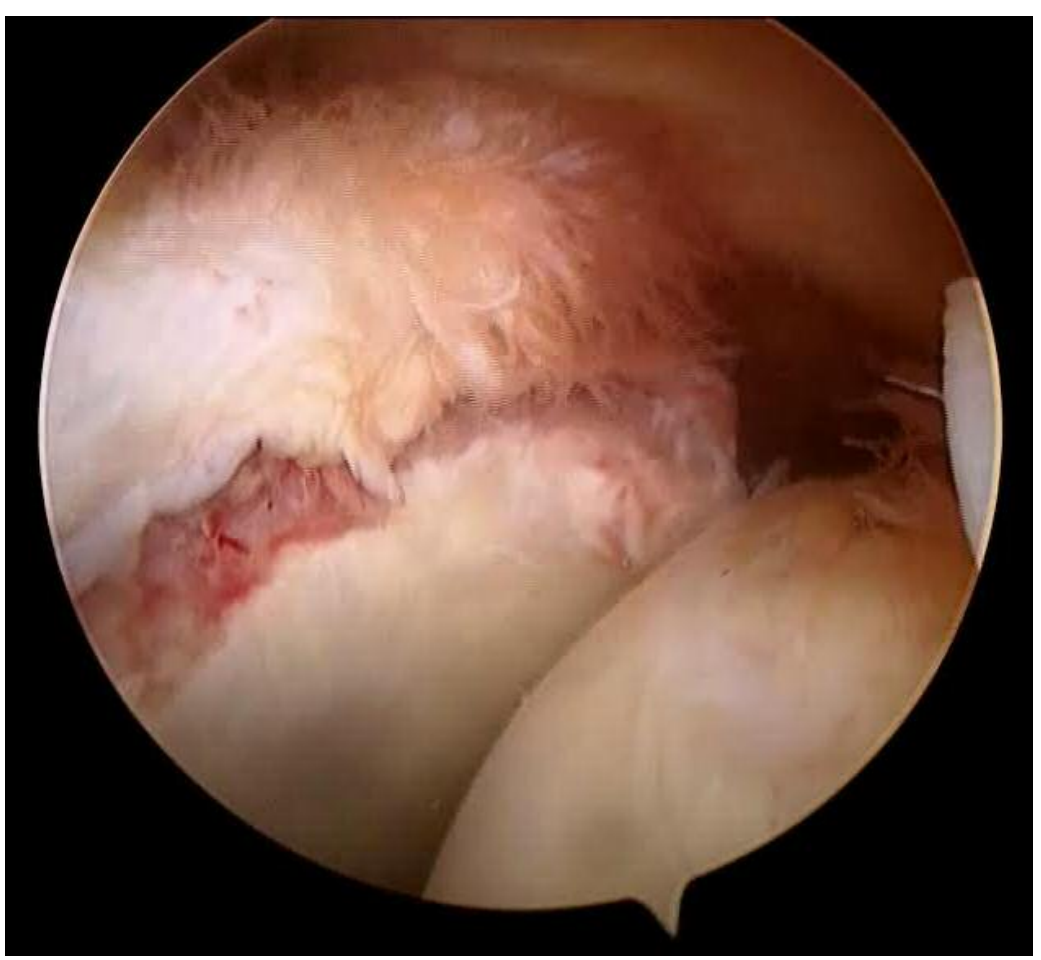

Figure 1. Arthroscopic assessment of the lesions. 


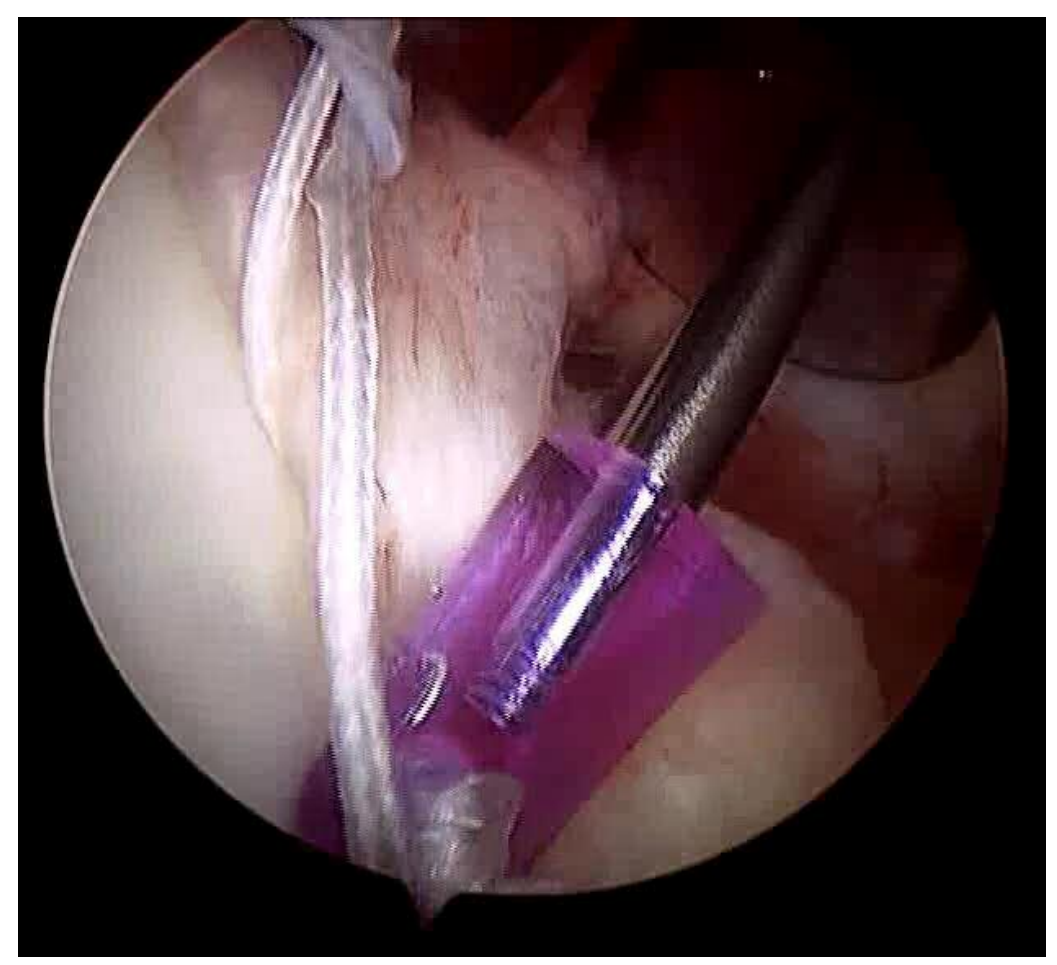

Figure 2. Fixation of the lesion with anchor.

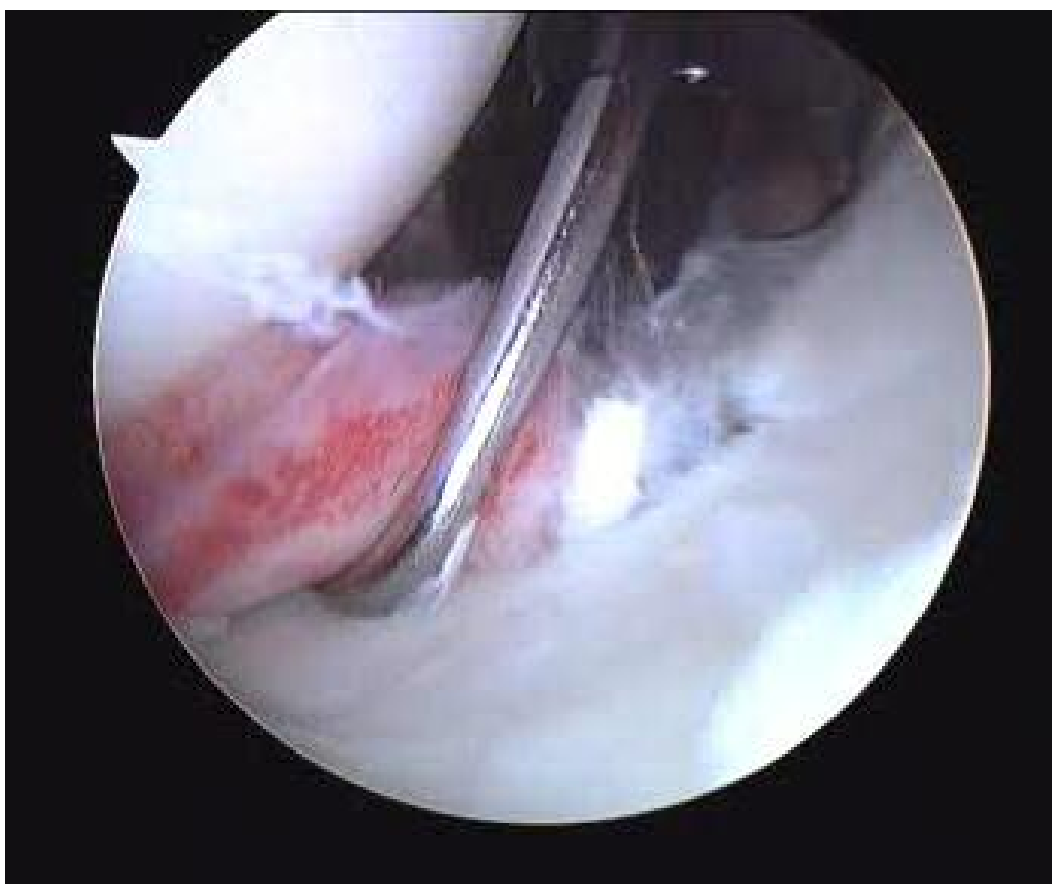

Figure 3. Final evaluation after fixation.

on the Rowe scores, the results in the open surgery group were excellent in 12 patients, good in 8 patients and poor in one patient.

In the arthroscopic repair group, the results were excellent in 21 patients and good in 4 patients.

Pain severity was high in the first post-operative day. Moreover, the pain in post-operative period and dur- ing rehabilitation was lower in the first group (average VAS respectively $6.0 \pm 1.0$ and $3 \pm 1.0$ in the first group and $4 \pm 1.0$ and $1 \pm 1.0$ in the second).

Shoulder range of motion was evaluated by comparison with the healthy shoulder and was determined according to the Rowe scoring. When the arms were positioned in the lateral position, loss of external rota- 
tion in the open surgery group was $20^{\circ}$ in one patient, $10^{\circ}$ in eight patients, and $5^{\circ}$ in 12 patients. In the arthroscopic repair group, loss of external rotation was seen only in 4 patients, being $10^{\circ}$ in 3 patients and $5^{\circ}$ in 1 patient.

Patients treated arthroscopically are returned before to sporting activities. In fact, patients underwent open surgery have recovered a full activity after eight weeks, instead the arthroscopic group in six $^{9}$.

\section{Discussion}

Dislocation mechanism consists of a violent movement of external rotation and abduction. It can result from contact sports between athletes (football, wrestling, rugby) or from a fall (motorcycle and cycling $)^{10}$. One of the most worrying problems is the recurrence of the dislocation, which is inversely proportional to the increase in age, with directly proportional associated lesions and risk of complications. Shoulder instability is a recurrent condition in various sports, but with different incidences, especially with pathological and clinical-pathological characteristics, age and sport type specific ${ }^{11,12}$. In literature, practice of an "overhead" or a "forced overhead" sport was the only risk factor identified of not returning to the same sport at a level that was similar or better following surgery ${ }^{1}$. Bankart repair, open or arthroscopically performed, has also been promoted in competitive athletes while several reports have described return to the same sport ranging from 71 to $98 \%{ }^{13,14}$ largely depending on the type of sports. In particular, Gerometta et al. described that arthroscopic Bankart repair showed favourable results to open repairs: most of the patients returned to the same or higher level of sports after a mean of 9.8 months postoperatively while male and less frequent dislocators $(<6$ dislocations) returned significantly faster to their sport and to their preinjury level than female and frequent dislocators (> 10 dislocations), respectively ${ }^{15}$. Petrera et al. ${ }^{16}$ and Milchteim et al. ${ }^{17}$ already showed how arthroscopic treatment of shoulder instability represents today not only a valid alternative to open surgery, but absolutely the first choice of treatment, because an arthroscopic repair restores the glenohumeral structures more precisely, reducing bleeding time and infections risk. Furthermore, Wellmann et al. ${ }^{18}$ demonstrated that arthroscopic surgery for shoulder instability leads to better functional results, especially in a shorter time.

Shoulder arthroscopy also has a diagnostic value, which is lost in open surgery, because arthroscopy allows to assess directly the presence of any lesions, respecting the joint anatomy, unlike the open technique ${ }^{19}$.

The advantages of arthroscopic treatment are the absence of iatrogenic muscle damage, lower risk of scar tissue adhesions, less invasive surgery, less postoperative pain, less extrarotation loss, and it also improves a diagnostic evaluation ${ }^{20}$. However, careful planning is needed to exclude patients with con- traindications such as severe bone defects in the glenoid (over $30 \%$ of its inferior diameter), engaging Hill Sachs lesions, and "Bony" Bankart lesions: in these cases open surgery is indicated ${ }^{21}$. In fact, some Authors showed that the Bankart arthroscopic procedure seems to be associated with a significant risk of recurrence in athletes: these failures appear to be due to the presence of bone loss 22 . The open surgery has a role only in the case of previous diseases and in case of recurrence after arthroscopy ${ }^{23}$. According to our observations, although the follow-up isn't long enough, the arthroscopic surgical approach appears to be excellent in shoulder instability management and it is therefore very beneficial. Arthroscopic treatment for shoulder instability had represented an alternative to open surgery ${ }^{24}$, but nowadays the literature, even if it is still controversial, showed that it is the primary indication. Recently Blonna et al. stated that arthroscopic stabilization using anchors provided better return to sport and subjective perception of the shoulder compared with the open Bristow-Latarjet procedure in the population studied: after a mean follow-up of 5.3 years patients who underwent arthroscopic Bankart repair obtained better results in terms of return to sport and ROM in the throwing position ${ }^{3}$. The arthroscopic technique achieves better functional results, in less time, and with a better cosmetic result. These needs are evident even more in professional athletes, because external pressures about recovery times and rapid return to high performance game weigh on them. Therefore in the case of professional athletes, it is not possible to enjoy a long period for conservative treatment, but it is necessary to resort quickly to the surgery, if the surgical indication exists. It is useful to do a pre and post-operative MRI evaluation, in order to ensure the correct placement of the anchors. It is also necessary to return as quickly as possible at sport activity through an intensive and specific rehabilitation ${ }^{25,26}$, performed several times during the day, based on sport-specific movements. In conclusion, according to our experience, arthroscopic surgical approach appears to be excellent in shoulder instability management, reducing recovery time, allowing a faster return to sport, with less pain, if compared with open surgery.

\section{Conflict of interest}

The Authors declare that they have no conflict of interest related to the publication of this manuscript.

\section{References}

1. Cho NS, Hwang JC, Rhee YG. Arthroscopic stabilization in anterior shoulder instability: collision athletes versus noncollision athletes. Arthroscopy. 2006;22:947-953.

2. Bessière $C$, Trojani $C$, Carles M, Mehta SS, Boileau P. The open latarjet procedure is more reliable in terms of shoulder stability than arthroscopic bankart repair. Clin Orthop Relat Res. 2014;472(8):2345-2351.

3. Blonna D, Bellato E, Caranzano F, Assom M, Rossi R, Castol- 
di F. Arthroscopic Bankart Repair Versus Open Bristow-Latarjet for Shoulder Instability: A Matched-Pair Multicenter Study Focused on Return to Sport. Am J Sports Med. 2016; pii: 0363546516.

4. Padulo J, Oliva F, Frizziero A, Maffulli N. Muscles, Ligaments and Tendons Journal - Basic principles and recommendations in clinical and field Science Research: 2016 Update. MLTJ. 2016;6(1):1-5.

5. De Coninck T, Ngai SS, Tafur M, Chung CB. Imaging the Glenoid Labrum and Labral Tears. Radiographics. 2016;36 (6):1628-1647.

6. Gibson J, Kerss J, Morgan C, Brownson P. Accelerated rehabilitation after arthroscopic Bankart repair in professional footballers. Shoulder Elbow. 2016;8(4):279-286.

7. McMenamin D, Koulouris G, Morrison WB. Imaging of the shoulder after surgery. Eur J Radiol. 2008;68(1):106-119.

8. Edouard P, Beguin L, Fayolle-Minon I, Degache F, Farizon F, Calmels P. Relationship between strength and functional indexes (Rowe and Walch-Duplay scores) after shoulder surgical stabilization by the Latarjet technique. Ann Phys Rehabil Med. 2010;53(8):499-510.

9. Salini V, Colucci C, Palmieri D, Croce G, Orso CA. The arthroscopic treatment of the post-traumatic shoulder instability. Medicina dello Sport. 2005;58(2):125-132.

10. Pastor MF, Smith T, Struck M, Wellmann M. Stability versus mobility of the shoulder. Biomechanical aspects in athletes. Orthopade. 2014;43(3):209-214.

11. Yamamoto N, Kijima H, Nagamoto $\mathrm{H}$, et al. Outcome of Bankart repair in contact versus non-contact athletes. Orthop Traumatol Surg Res. 2015;101(4):415-419.

12. Uchiyama Y, Hamada K, Miyazaki S, Handa A, Fukuda H. Neer modified inferior capsular shift procedure for recurrent anterior instability of the shoulder in judokas. Am J Sports Med. 2009;37(5):995-1002.

13. Castagna A, Markopoulos N, Conti M, Delle Rose G, Papadakou E, Garofalo R. Arthroscopic bankart suture-anchor repair: radiological and clinical outcome at minimum 10 years of follow-up. Am J Sports Med. 2010;38(10): 2012-2016.

14. O'Neill DB. Arthroscopic Bankart repair of anterior detachments of the glenoid labrum. A prospective study. J Bone Joint Surg Am. 1999;81(10):1357-1366.

15. Gerometta A, Rosso C, Klouche S, Hardy P. Arthroscopic
Bankart shoulder stabilization in athletes: return to sports and functional outcomes. Knee Surg Sports Traumatol Arthrosc. 2016;24(6):1877-1883.

16. Petrera M, Patella V, Patella S, Theodoropoulos J. A metaanalysis of open versus arthroscopic Bankart repair using suture anchors. Knee Surg Sports Traumatol Arthrosc. 2010;18 (12):1742-1747.

17. Milchteim C, Tucker SA, Nye DD, et al. Outcomes of Bankart Repairs Using Modern Arthroscopic Technique in an Athletic Population. Arthroscopy. 2016;32(7):1263-1270.

18. Wellmann M, Bobrowitsch E, Khan N, et al. Biomechanical effectiveness of an arthroscopic posterior bankart repair versus an open bone block procedure for posterior shoulder instability. Am J SportsMed. 2011;39(4):796-803.

19. Nourissat G, Radier C, Aim F, Lacoste S. Arthroscopic classification of posterior labrum glenoid insertion. Orthop Traumatol Surg Res. 2014;100(2):167-170.

20. Zhang AL, Montgomery SR, Ngo SS, Hame SL, Wang JC, Gamradt SC. Arthroscopic versus open shoulder stabilization: current practice patterns in the United States. Arthroscopy. 2014;30(4):436-443.

21. Cole BJ, Millett PJ, Romeo AA, et al. Arthroscopic treatment of anterior glenohumeral instability: indications and techniques. Instr Course Lect. 2004;53:545-558.

22. Neyton L, Young A, Dawidziak B, et al. Surgical treatment of anterior instability in rugby union players: clinical and radiographic results of the Latarjet-Patte procedure with minimum 5-year follow-up. J Shoulder Elbow Surg. 2012;21:1721-1727.

23. De Giorgi S, Garofalo R, Tafuri S, Cesari E, Rose GD, Castagna A. Can arthroscopic revision surgery for shoulder instability be a fair option? Muscles Ligaments Tendons $\mathrm{J}$. 2014;4(2):226-231.

24. Freedman KB, Smith AP, Romeo AA, Cole BJ, Bach BR Jr. Open Bankart repair versus arthroscopic repair with transglenoid sutures or bioabsorbable tacks for Recurrent Anterior instability of the shoulder: a meta-analysis. Am J Sports Med. 2004;32(6):1520-1527.

25. Sicuri C, Porcellini G, Merolla G. Robotics in shoulder rehabilitation. Muscles Ligaments Tendons J. 2014;4(2):207-213.

26. Rooney SI, Loro E, Sarver JJ, et al. Exercise protocol induces muscle, tendon, and bone adaptations in the rat shoulder. Muscles Ligaments Tendons J. 2015;4(4):413-419. 